\title{
Improved MicroED structures by cryo-FIB milling
}

\author{
M Martynowycz ${ }^{1}$ \\ ${ }^{1}$ HHMI/UCLA, Los Angeles, CA \\ michael.martynowycz@gmail.com
}

Microcrystal electron diffraction is an electron cryo-microscopy method that determines crystal structures from vanishingly small crystals. The strong interaction between high energy electrons and matter limits the size of crystals to those typically thinner than perhaps 500 nanometers. Though some crystals will naturally form crystals small enough for MicroED, many will end up too large for MicroED and too small for synchrotron X-ray diffraction experiments. We demonstrate the combination of scanning electron microscopy (SEM) and cryogenic focused ion-beam (FIB) milling of protein microcrystals. Our approach results in ideally thick specimens with better crystallographic statistics, and opens MicroED to entirely new experiments. Here, we present the methods, rationale, and recent results using this approach.

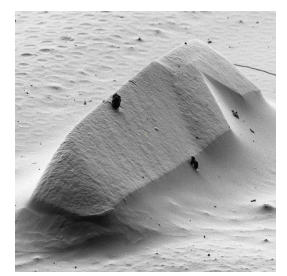

Figure 1

Acta Cryst. (2020). A76, a165 